\title{
Millimeter-Scale Contact Printing of Aqueous Solutions Using a Stamp Made Out of Paper and Tape
}

\section{Citation}

Cheng, Chao-Min, Aaron D. Mazzeo, Jinlong Gong, Andres W. Martinez, Scott T. Phillips, Nina Jain, and George M. Whitesides. 2010. Millimeter-scale contact printing of aqueous solutions using a stamp made out of paper and tape. Lab on a Chip 10(23): 3201-3205.

\section{Published Version}

doi:10.1039/C004903D

\section{Permanent link}

http://nrs.harvard.edu/urn-3:HUL.InstRepos:9871958

\section{Terms of Use}

This article was downloaded from Harvard University's DASH repository, and is made available under the terms and conditions applicable to Open Access Policy Articles, as set forth at http:// nrs.harvard.edu/urn-3:HUL.InstRepos:dash.current.terms-of-use\#OAP

\section{Share Your Story}

The Harvard community has made this article openly available.

Please share how this access benefits you. Submit a story.

\section{Accessibility}




\section{Millimeter-Scale Contact Printing of Aqueous Solutions using a Stamp Made out of Paper and Tape}

Chao-Min Cheng, Aaron D. Mazzeo, Jinlong Gong, ${ }^{\#}$ Andres W. Martinez, ${ }^{\#}$ Scott T. Phillips, Nina Jain, and George M. Whitesides*

Department of Chemistry \& Chemical Biology, Harvard University, Cambridge, MA 02138, U.S.A.

*Corresponding author; E-mail: gwhitesides@gmwgroup.harvard.edu

${ }^{\#}$ These authors contributed equally to this work. 


\begin{abstract}
This communication describes a simple method for printing aqueous solutions with millimeter-scale patterns on a variety of substrates using an easily fabricated, paperbased microfluidic device (a paper-based "stamp") as a contact printing device. The device is made from inexpensive materials, and it is easily assembled by hand; this method is thus accessible to a wide range of laboratories and budgets. A single device was used to print over 2500 spots in less than three minutes at a density of 16 spots per square centimeter. This method provides a new tool to pattern biochemicals - reagents, antigens, proteins, and DNA — on planar substrates. The accuracy of the volume of fluid delivered in simple paper-to-paper printing is low, and although the pattern transfer is rapid, it is better suited for qualitative than accurate, quantitative work. By patterning the paper to which the transfer occurs using wax printing or an equivalent technique, accuracy increases substantially.
\end{abstract}


This paper describes a technique for printing patterns of aqueous solutions using three-dimensional, microfluidic, paper-based analytical devices (3D- $\mu$ PADs) as "stamps" (e.g. pattern-transfer agents). This technique is a useful addition to the range of microfluidic devices that can be constructed using paper, and has applications in adding reagents to other paper-based microfluidic devices and in patterning reagents at millimeter scale, on paper, and on other flat substrates. We and others have developed various contact printing methods to pattern bacteria, ${ }^{1}$ self-assembled monolayers (SAMs), ${ }^{2,3}$ polymers, ${ }^{4}$ proteins,${ }^{5,6}$ peptides $,{ }^{7} \mathrm{DNA},{ }^{8}$ biomolecules for cell culture, ${ }^{9}$ microorganisms, ${ }^{10}$ and metals ${ }^{11}$ on a variety of substrates with micro-scale resolution. Even though these methods could be useful in paper-based microfluidics, they are often expensive, and their resolutions are greater than that required for paper-based devicestypically millimeter-scale patterns designed to be read by eye, or using a cell-phone camera — and may have other weaknesses: they deliver limited quantities of reagents, require frequent inking, and require materials of construction that may not be broadly available. Inkjet printers provide an excellent method for patterning materials on paper and other thin and flexible materials, and they have been demonstrated in applications with $\mu \mathrm{PADs}^{12}$; however, these methods typically require customization of the printer and inks, and are probably better suited for large-scale manufacturing than for prototyping.

This paper-based system we describe here for printing has four useful characteristics: (i) The flexibility in design and fabrication of the stamps allows for rapid prototyping of printing devices; a completely new device can be fabricated in less than two hours. (ii) The paper-based stamp is easily connected to fluid reservoirs that automatically supply the printing area with fresh ink through paper-based microfluidic 
channels; most printing methods require periodic re-inking of the stamp. (iii) Since each fluid reservoir can hold a different "ink", it is straightforward to print several (in this work, up to four) different solutions in a single printing step. (iv) The technology requires little in the way of infrastructure and is appropriate for use in resource-limited environments. The disadvantages of this method are that, in its current form, the resolution is limited to the millimeter scale, there is variation in quantity of reagent delivered, and in the shape of the printed pattern, which can be high unless other constraints are introduced. The system can also only be used with aqueous solutions, or other solutions that do not wick uncontrollably in $\mu$ PADs.

We fabricated the stamps by stacking layers of patterned paper and double-sided adhesive tape as described previously, and detailed in the Supplementary Information (Figure 1A). ${ }^{12-14}$ Stacking layers of patterned paper and double-sided adhesive tape yielded three-dimensional paper-based microfluidic stamps for printing. Aqueous solutions introduced at the fluid reservoirs wicked through the entire paper-based device to form the desired array of spots. It takes $\sim 15$ minutes to assemble a stamp manually from its necessary components. The bottom layer of the device consists of a piece of paper patterned with the shapes to be printed. Each shape on the bottom layer of the stamp is connected to fluid reservoirs on the top layer of the stamp through channels patterned into the middle layers of the device. We designed the devices with fluid reservoirs outside of the printing area, so that we could apply pressure to the printing area without affecting the reservoirs. Attaching a piece of plexiglass with inlet holes to the top of the device provided fluid reservoirs and a rigid support for the stamp (Figure 1A). A wooden handle attached to the plexiglass facilitated manipulation of the stamp (Figure 
1B). Inserting $200-\mu \mathrm{L}$ pipette tips into the holes in the plexiglass increased the capacity of the fluid reservoirs (Figure 1C).

To prime the device for printing, $\sim 20 \mu \mathrm{L}$ of solution were allowed to wick from the fluid reservoirs to the bottom of the device. Once the solution reached the bottom of the device ( $\sim 5$ minutes after adding the solution to the fluid reservoir), the device was ready for printing (Figure 1D). When the device is pressed against a substrate, only the area containing the patterns to be printed comes into contact with the substrate.

We printed a variety of 'inks' on different substrates to establish the generality of the method. Wetting of the substrate, and capillary action, allowed inks to transfer from the paper-based stamp to the substrate. We printed aqueous solutions of small molecules, proteins, and nucleic acids in different shapes and patterns on a wide variety of substrates including paper, glass, polystyrene (PS), nitrocellulose (NC) membranes, cellulose acetate (CA) membranes, hydrophilic polyvinylidene fluoride (PVDF) membranes and thin-layer chromatography (TLC) plates made with silica gel (as shown in Supplementary Information). Both porous, hydrophobic substrates and non-porous, hydrophilic substrates accepted ink, but the resolution was lower on the latter than on the former.

The frequency with which prints can be made manually using this type of stamp is around 15 per minute, and we have successfully used a single stamp to print over 500 patterns with no signs of deterioration. In systems in which the stamping area connects to fluid reservoirs that supply "ink", we have made more than 40 pattern transfers before refilling the reservoirs. The fluid reservoirs can hold different solutions, so that multiple solutions can be printed simultaneously. 
Figure 1. A paper-based stamp for printing on paper. A) Schematic representation of the layers of paper and tape in a paper-based stamp. The fluid reservoirs are offset from the printing area so that pressure can be applied to the printing area without affecting the reservoirs. The fluidic path in this device starts at the fluid reservoir, goes through holes in double-sided tape, follows channels patterned in a sheet of paper, goes through four more layers of double-sided tape, and follows patterned paths on four more pieces of paper before exiting the device. B) Side-view of a paper-based stamp. C) Side-view of four reservoirs of this paper-based stamp; these fluid reservoirs provide aqueous solutions for printing. Pipette tips can also be connected to these reservoirs to provide a continuous feed of fluid for printing (red arrow: pipette tips). D) Bottom view of a paper-based stamp filled with aqueous solutions of dyes. E) Array of spots printed on Whatman chromatography paper No. 1 using the device shown in (D). F) Array of spots printed on patterned Whatman chromatography paper No. 1 using the device shown in (D). In this case, the stamp transferred fluid to a set of empty wells on a piece of paper patterned by photolithography into arrays of wells that matched the pattern of the stamp. A similar method is described in more detail in Figure 3. 
Figure 1.

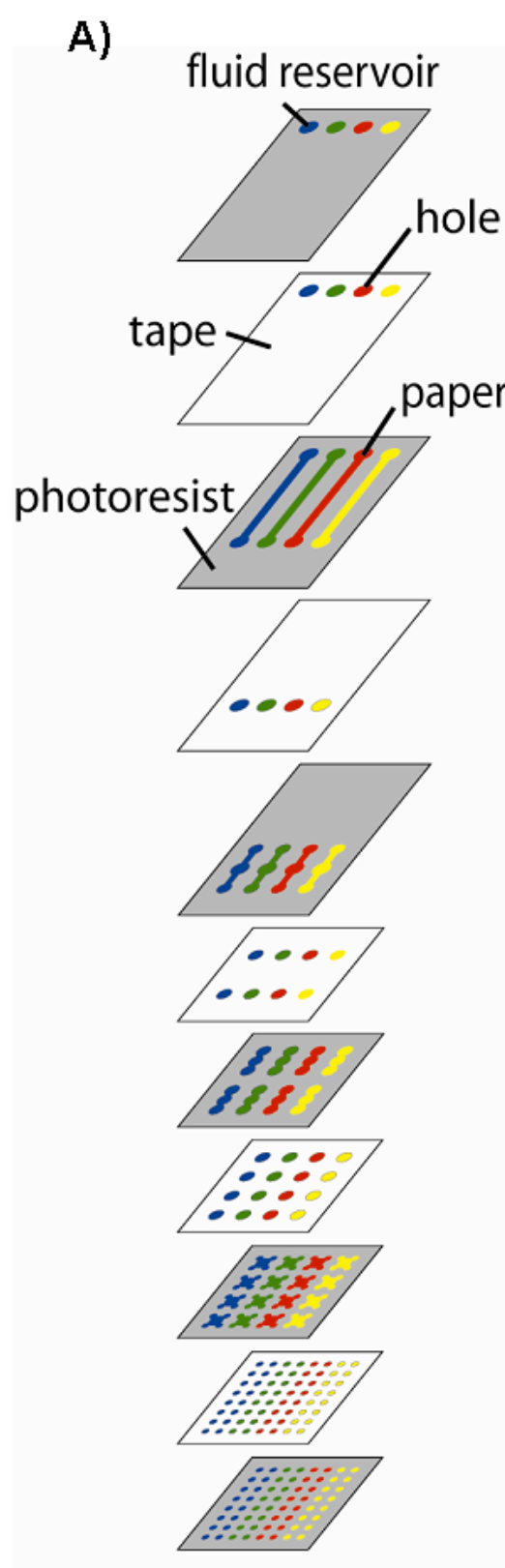

B)

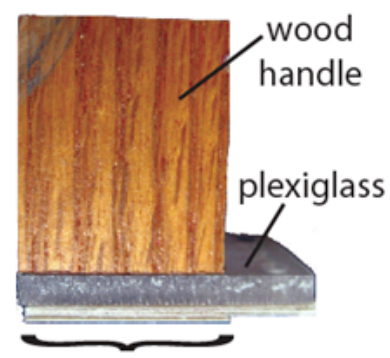

stamping area pipette tip

C)

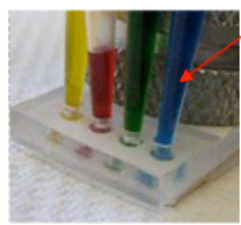

D)

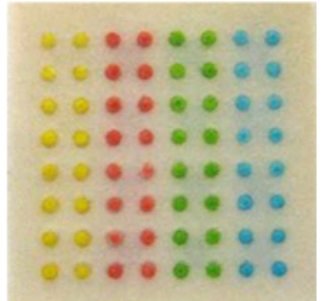

E)

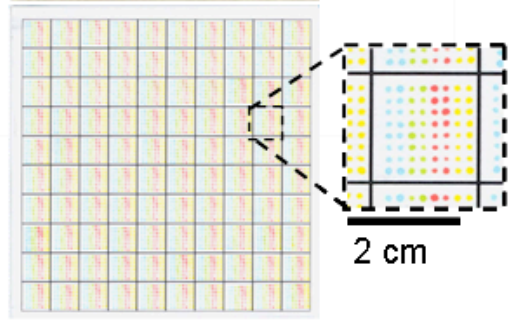

F)

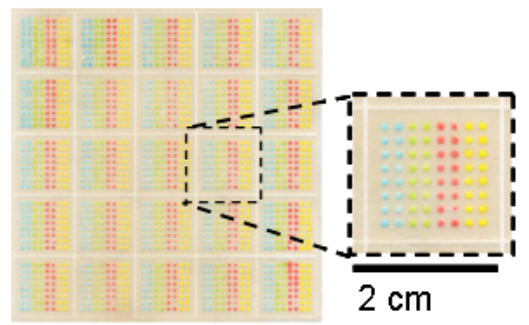


The stamps can print arrays of spots at densities $\left(25 \mathrm{spots} / \mathrm{cm}^{2}\right)$ comparable to many array-based formats. For example, these densities are similar to those in 384-well formats $\left(9 \mathrm{~mm}^{2} /\right.$ well or $\left.11 \mathrm{wells} / \mathrm{cm}^{2}\right)$ and could easily be designed to fit into devices designed for this format. ${ }^{15}$

\section{The Quality of Printing}

To evaluate the performance of paper-based stamps, we printed patterns of circular spots on Whatman chromatography paper No. 1 using low-molecular-weight, water-soluble dyes [Allura red (red), Erioglaucine (blue), Tartrazine (yellow), and Tartrazine (green)] (Figure 1E \& 2A) and on patterned Whatman chromatography paper No. 1 (Figure 1F), respectively. The resulting patterns demonstrate the capability of the method for printing small molecules, and since the patterns are easily visible, they can be used to evaluate the performance of the method (Figure 2). The pattern shown in Figure 2A contains an array of 64 spots each with a nominal diameter of $1 \mathrm{~mm}$ and an area of $0.79 \mathrm{~mm}^{2}$. With $20 \mu \mathrm{L}$ of sample added to each fluid reservoir, we were able to print 45 patterns in 3 minutes. Each impression took approximately 4 seconds to complete $(\sim 2$ seconds to align the stamp with the substrate and $\sim 2$ seconds of contact time between the stamp and the paper). The measured area of the printed spots was $1.0 \pm 0.4 \mathrm{~mm}^{2}$ (average \pm standard deviation, $N=1601)$. The calculated mean diameter of the spots $(\pi / 4 \times$ square of the diameter is the area) was $1.1 \pm 0.2 \mathrm{~mm}$. 
Figure 2. Patterns printed with a paper-based stamp on Whatman Chromatography paper No. 1. A) Array of 64 circular spots nominally $1 \mathrm{~mm}$ in diameter. B) Histogram with fitted normal distribution of the measured diameters for the circular spots shown in (A). For the number of measured areas in the processed image $(\mathrm{N}=1601)$ within the specified range of areas $\left(0.08 \mathrm{~mm}^{2}\right.$ to $\left.3.1 \mathrm{~mm}^{2}\right)$, the mean is $1.0 \pm 0.4 \mathrm{~mm}^{2}$. See supplementary information for details concerning the method for measuring areas. C) Array of large shapes. D) Array of small shapes. The solid lines on the right plane show the original design of patterns. The printed results are slightly larger than the patterns in the stamp because of the lateral spreading of fluid on the paper. We printed black lines on the paper substrate using a laser printer to help align the paper-based stamp to the substrate. 
Figure 2.

A)

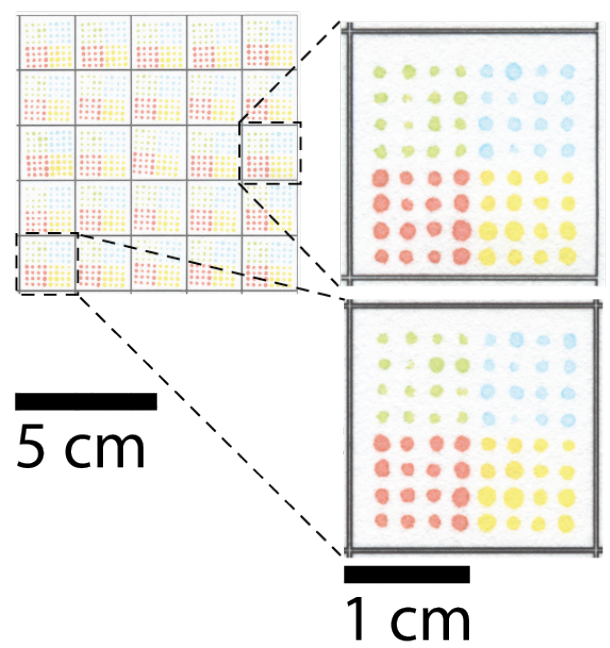

B)

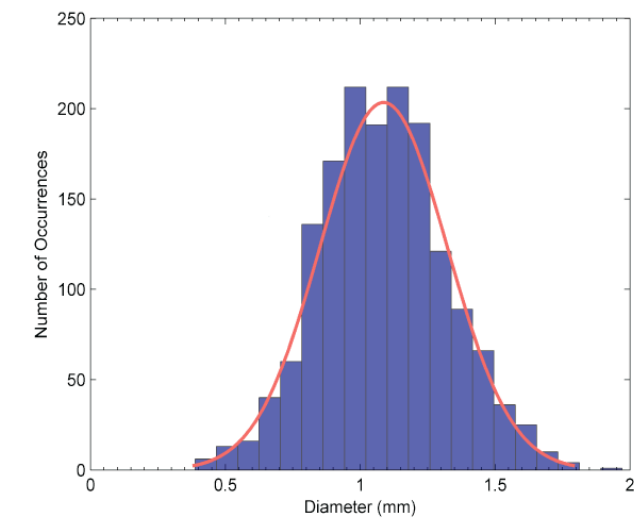

C)

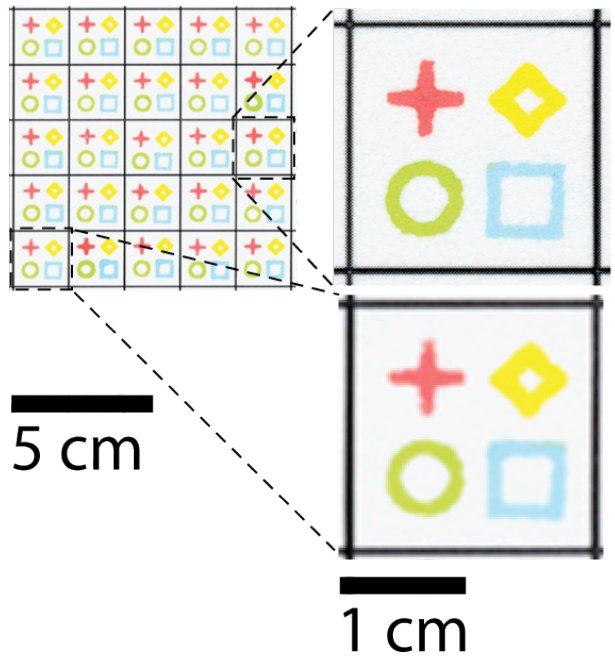

D)

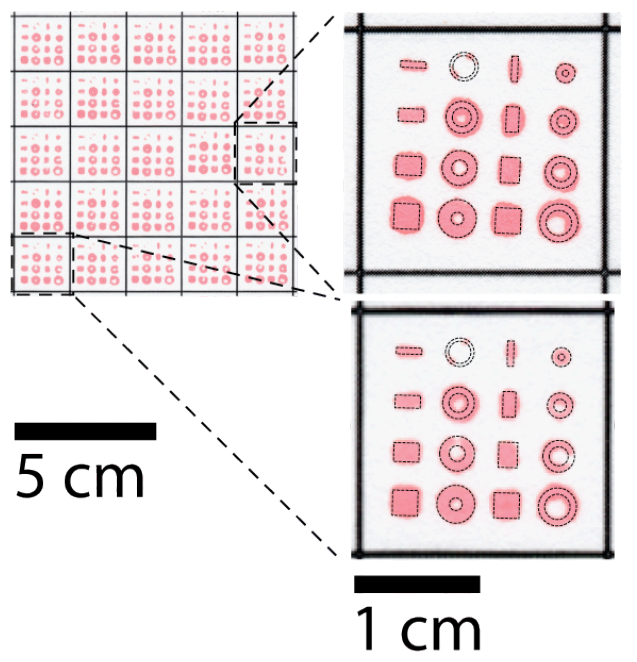


The size of the printed spots is generally larger than the pattern on the stamp because the aqueous solutions wicked laterally on hydrophilic substrates (e.g., paper) after application. Figure 2B shows a histogram depicting the distribution of areas measured in Figure 2A. The supplementary information outlines the automated method we used to measure the areas of the spots. For one sheet of arrays of spots (Supplementary Figure 1A), the average measured area for the spots was $1.0 \pm 0.4 \mathrm{~mm}^{2}$ with $\mathrm{N}=6166$ (calculated mean diameter was $1.1 \pm 0.2 \mathrm{~mm}^{2}$ with $\mathrm{N}=6166$ ). For another similarly printed sheet (Supplementary Figure 1B), the average measured area for the spots was $1.0 \pm 0.4 \mathrm{~mm}^{2}$ with $\mathrm{N}=6394$ (calculated mean diameter was $1.1 \pm 0.2 \mathrm{~mm}^{2}$ with $\mathrm{N}=6394$ ). We estimate that each spot consumed $\sim 30 \mathrm{~nL}$ of solution, and that the device delivered $\sim 2 \mu \mathrm{L}$ of solution per square centimeter of printed surface area.

The coefficient of variation (standard deviation divided by the mean) for the measured areas of the spots was $\sim 40 \%$ ( $18 \%$ for the calculated diameters). Fluctuations in the quality of the printing may result from variations in porosity of the substrate, cleanliness of the substrate, relative differences in hydrophobicity/hydrophilicity between the substrate and applied liquids, contact time, time between imprints, applied pressure, and flow of fluid through the network of channels in the device. We have not made a serious engineering effort to reduce the variation at this stage of development of the device. For longer contact times between the stamp and the substrate, capillary action will draw more fluid from the stamp to the substrate. For variations in time between imprints, different transferrable quantities of fluid might be available at the head of the stamp. With long periods of time between imprints, fluid can accumulate on the head of the stamp. With periods of time between imprints (less than 1 second) shorter than the 
time to refill each printing area, we have observed that the head of the stamp becomes depleted in ink. This results in fluid not being transferred to the substrate in some locations. Paper-based microfluidic channels and vias can vary in their fluidic resistance (i.e., the time required for fluid to fill all the regions on the stamp to a steady state value can vary from spot to spot). This variation may also contribute to variations in the areas of different spots printed simultaneously. Because the experiments in this work were manual, we did not precisely control the pressure, and the applied force between imprints.

The large systematic variation in the quantity of fluid transferred during printing will limit the utility of this type of contact printing for experiments and assays that require accurate and reproducible transfer of fluids for quantitative analysis. Paper-based contact printing may therefore be most useful in applications in which the response is semiquantitative or binary (e.g., presence or lack of a specific antigen). To what extent reproducibility can be improved by optimizations in design, fabrication, and operation remains to be determined.

\section{Printing into Pre-Defined Areas}

One of the factors contributing to spot-to-spot variation is variability in the wicking (both in the sheet being printed, and in the channels supplying the fluid). At least one factor - the areas being wet-is under our control. By using wax printing ${ }^{16}$ to define printed, hydrophilic areas of approximately $1 \mathrm{~mm}^{2}$ in the substrate receiving transferred ink, we reduced the coefficient of variation for the measured areas from $40 \%$ (Figure 2B) to $21 \%$ (Figure 3B), which corresponds to a reduction in the coefficient of variation from $18 \%$ to $11 \%$ for the calculated diameters. For substrates with larger printed areas of approximately $3 \mathrm{~mm}^{2}$ and contact times of $10 \mathrm{~s}$ to transfer the ink from the paper-based 
stamp, we further reduced the coefficient of variation for the measured areas to $11 \%(6 \%$ for the calculated diameters).

To study the effects of size and contact time on the reproducibility of printed spots, we printed water-soluble dye (Allura red) into the hydrophilic circular regions with two nominally different diameters and three different contact times for holes of each size. For the smaller sets of printed spots (diameters of $\sim 1 \mathrm{~mm}$ ), the contact times were approximately 2, 3, and $4 \mathrm{~s}$ (low, medium, and high). For the larger sets of printed spots (diameters of $\sim 2 \mathrm{~mm}$ ), the contact times were approximately 2,5 , and $10 \mathrm{~s}$ (low, medium, and high). The time between printing steps was $3 \mathrm{~s}$, except in cases when refilling the reservoirs was necessary.

For contact times of $4 \mathrm{~s}$ with the smaller spots, the calculated mean diameter was $0.9 \pm 0.1 \mathrm{~mm}$ with $\mathrm{N}=400$ (coefficient of variation of $11 \%$ ); for contact times of $10 \mathrm{~s}$ with the larger spots, the calculated mean diameter was $2.0 \pm 0.1 \mathrm{~mm}$ with $\mathrm{N}=400$ (coefficient of variation of 6\%). As shown in Figure 3C, the coefficient of variation for these sets of experiments was lowest when printing the larger spots with the longest contact time. For the larger spots, shorter contact times of 2 and $5 \mathrm{~s}$ correlated with higher coefficients of variation for the printed areas, which are a result of partial filling of the hydrophilic circular regions in the substrate. For the smaller spots, shorter contact times of 2 and $3 \mathrm{~s}$ did not correlate with higher coefficients of variation in both cases (coefficient of variation of $13 \%$ for a contact time of $2 \mathrm{~s}$; coefficient of variation of $9 \%$ for a contact time of $3 \mathrm{~s}$ ). However, the smaller printed spots with a contact time of $2 \mathrm{~s}$ resulted in more partially filled regions than those found in the substrates with smaller printed spots with contact times of 3 and $4 \mathrm{~s}$ (Figure 3B and Supplementary Figure 6C and D). 
Figure 3. Contact printing on pre-patterned Whatman Chromatography paper No. 1. We used the technique of wax-printing to pattern both the paper-based substrates receiving the water-soluble dye (Allura red) and the individual layers of patterned paper within the stamp. A) Printed spots with a mean diameter of $2.0 \pm 0.1 \mathrm{~mm}$ with $\mathrm{N}=400$ (coefficient of variation of $6 \%$ ) on a pre-patterned substrate. The accompanying histogram shows the distribution of the measured diameters, along with a fitted normal distribution. The contact time was $10 \mathrm{~s}$. B) Printed spots with a mean diameter of $0.9 \pm 0.1 \mathrm{~mm}$ with $\mathrm{N}=400$ (coefficient of variation of $11 \%$ ) on a pre-patterned substrate. The accompanying histogram shows the distribution of the measured diameters, along with a fitted normal distribution and a fitted extreme value distribution. The contact time was $4 \mathrm{~s}$. C) Bar chart for the measured mean diameters for printed spots of smaller diameter $(\sim 1 \mathrm{~mm})$ and larger diameter $(\sim 2 \mathrm{~mm})$ with low, medium, and high contact times $(2,3$, and $4 \mathrm{~s}$ for smaller spots; 2,5 , and $10 \mathrm{~s}$ for the larger spots). The error bars are \pm 1 standard deviation. 
Figure 3.
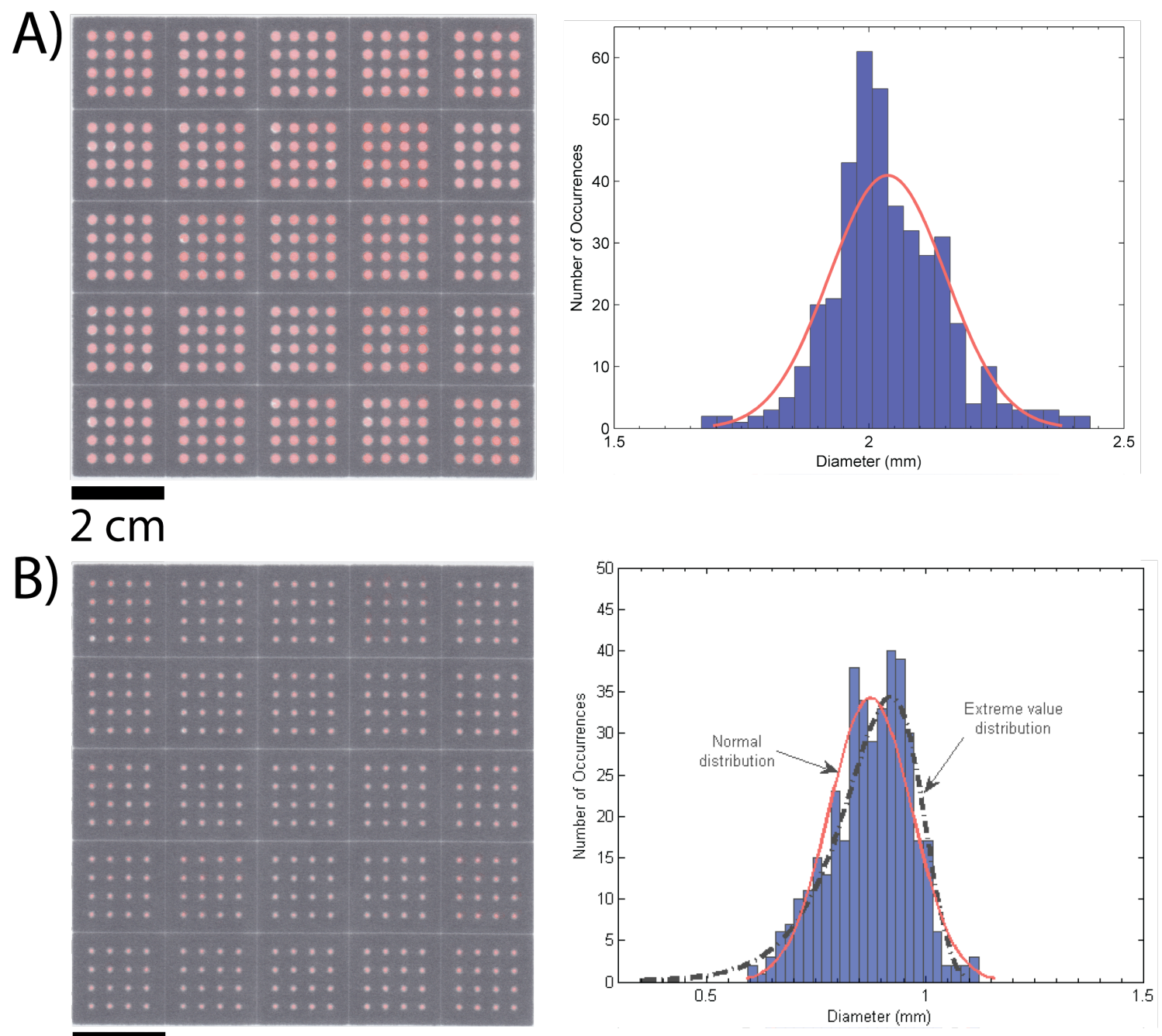

\section{$2 \mathrm{~cm}$}

C)

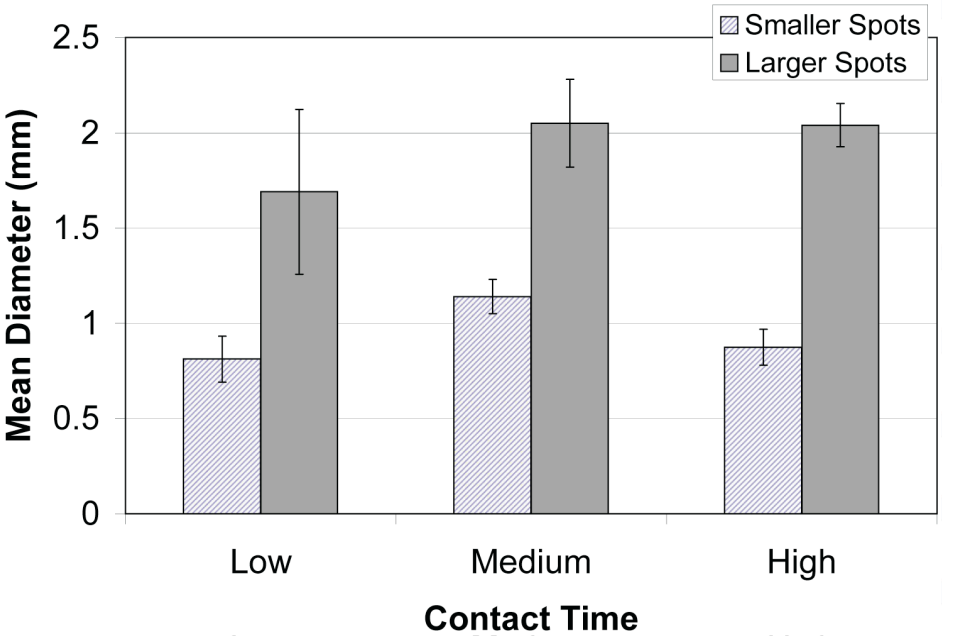


Paper-based stamps are not limited to printing circular spots. They can also be used to print simple shapes. Figure 2 and Supplementary Figure 5 show two sets of shapes printed on paper. Larger shapes (line width $\geq 1 \mathrm{~mm}$ ) can be printed with wellresolved patterns. We also tested our ability to print aqueous solutions of the small molecule dyes on other substrates: NC membranes, CA membranes, PVDF membranes, and silica gel (Supplementary Figure 5B).

Paper-based stamps provide a versatile and inexpensive system for printing repetitive patterns on different substrates. These stamps are capable of patterning large features ( $\geq 1 \mathrm{~mm})$, and transfer of ink from a stamp to pre-defined areas can improve the reproducibility of features. In the case of transferring ink to a pre-patterned substrate, increasing contact times can lower the variation of spotted areas by increasing the probability of completely filling pre-defined areas. The pre-patterned substrates and stamps are made from inexpensive materials (e.g., paper and tape) and can be prototyped rapidly, thus making this printing method ideal for preliminary experiments and accessible to a wide range of laboratories.

\section{ACKNOWLEDGEMEnTS}

This work was funded by the Bill \& Melinda Gates Foundation (\# 51308, "ZeroCost Diagnostics") and the MF3 Micro/Nano Fluidics Fundamentals Focus Center. 


\section{REFERENCES}

1 D. B. Weibel, A. Lee, M. Mayer, S. F. Brady, D. Bruzewicz, J. Yang, W. R. DiLuzio, J. Clardy and G. M. Whitesides, Langmuir, 2005, 21, 6436-6442.

2 J. Lahiri, E. Ostuni and G. M. Whitesides, Langmuir, 1999, 15, 2055-2060.

3 M. Mrksich, C. S. Chen, Y. Xia, L. E. Dike, D. E. Ingber and G. M. Whitesides, Proc. Natl. Acad. Sci. USA, 1996, 93, 10775-10778.

4 G. Csucs, R. Michel, J. W. Lussi, M. Textor and G. Danuser, Biomaterials, 2003, 24, 1713-1720.

5 H. B. Lu, J. Homola, C. T. Campbell, G. G. Nenninger, S. S. Yee and B. D. Ratner, Sens. Actu. B, 2001, 74, 91-99.

6 C.-M. Cheng and P. R. LeDuc, J. Am. Chem. Soc., 2007, 129, 9546-9547.

7 H. Zhang, J. N. Hanson Shepherd and R. G. Nuzzo, Soft Matter, 2010, 6, 2238-2245.

8 O. Akbulut, A. A. Yu and F. Stellaci, Chem. Soc. Rev., 2010, 39, 30-37.

9 I. Elloumi-Hannachi, M. Maeda, M. Yamato and T. Okano, Biomaterials, 2010 (in press).

10 C. Ingham, J. Bomer, A. Sprenkels, A. van den Berg, W. de Vos and J. van Hylckama Vlieg, Lab Chip, 2010, 10, 1410-1416.

11 H. Schmid, H. Wolf, R. Allenspach, H. Riel, S. Karg, B. Michel and E. Delamarche, Adv. Funct. Mater., 2003, 13, 145-153.

12 A. W. Martinez, S. T. Phillips, B. J. Wiley, M. Gupta and G. M. Whitesides, Lab Chip, 2008, 8, 2146-2150.

13 A. W. Martinez, S. T. Phillips and G. M. Whitesides, Proc. Natl. Acad. Sci. USA, 2008, 105, 19606-19611. 
14 A. W. Martinez, S. T. Phillips, G. M. Whitesides and E. Carrilho, Anal. Chem., 2010, 82, 3-10.

15 E. Carrilho, S. T. Phillips, S. J. Vella, A. W. Martinez and G. M. Whitesides, Anal. Chem., 2009, 81, 5990-5998.

16. E. Carrilho, A. W. Martinez and G. M. Whitesides, Anal. Chem., 2009, 81, 70917095. 